\section{depósito elevado para la Fiat}

PIER LUIGI NERVI, ingeniero

$581-23$

\section{sinopsis}

En la factoría Fiat, de Turín (Italia), se ha construido este depósito elevado, en forma de copa, de $2.000 \mathrm{~m}^{3}$ de capacidad $y$ unos $60 \mathrm{~m}$ de altura sobre el suelo.

Es de hormigón armado y paneles prefabricados, apoyándose sobre un disco de hormigón, una corona cilíndrica y contra

El fuste, de $8 \mathrm{~m}$ de diámetro, se ha construido con la ayuda de una serie de paneles, de superficie cilíndrica y sección en forma de $U$ con alas asimétricas, de $3 \mathrm{~cm}$ de espesor, que sirvieron de encofrado exterior en el hormigonado de las paredes, cuyo espeso" varía de $60 \mathrm{~cm}$ en la zona inferior a $50 \mathrm{~cm}$ en la superior.

La cubeta es un meridiano parabólico de revolución, constituido por paneles triangulares, formando anillos troncocónicos, que se utilizaron tambien como encofrado, apoyado sobre un entramado tubular en voladizo.

Encima de la cubeta se ha construido un mirador, de $25 \mathrm{~m}$ de diámetro, cubierto con una cúpula nervada esférica, soportada por montantes articulados que permiten movimientos radiales sin transmitir momentos fiectores al anillo superior de depósito, el cual, por estar lleno de agua, tiene temperatura
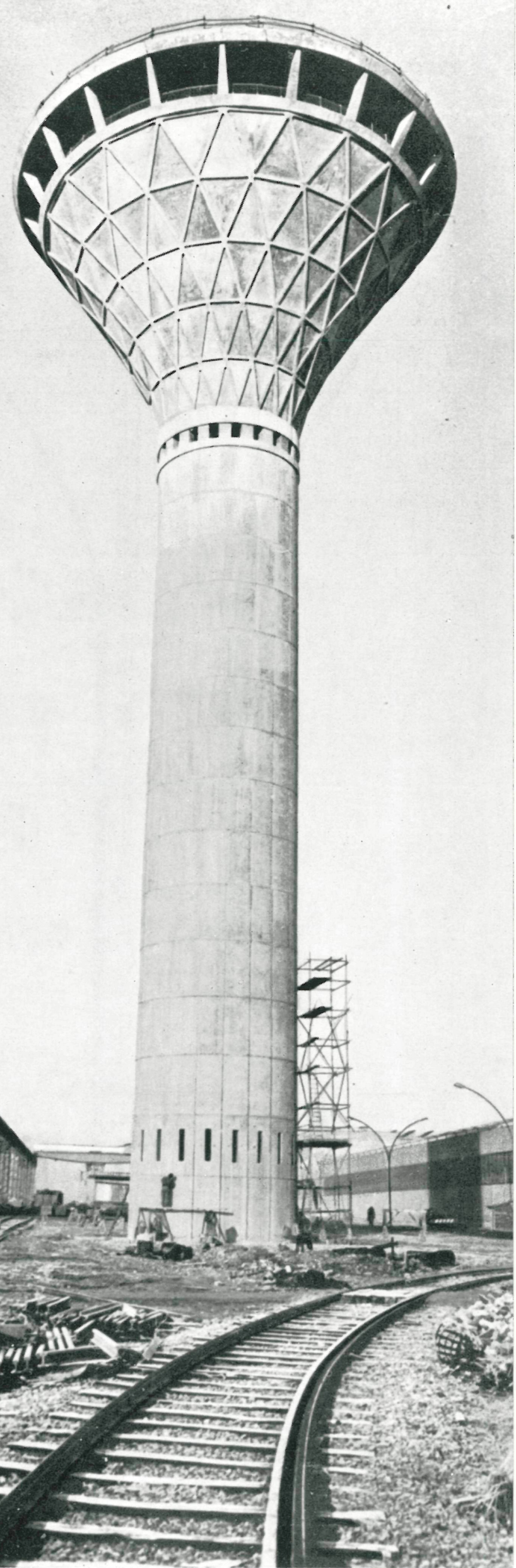


\section{sección vertical}

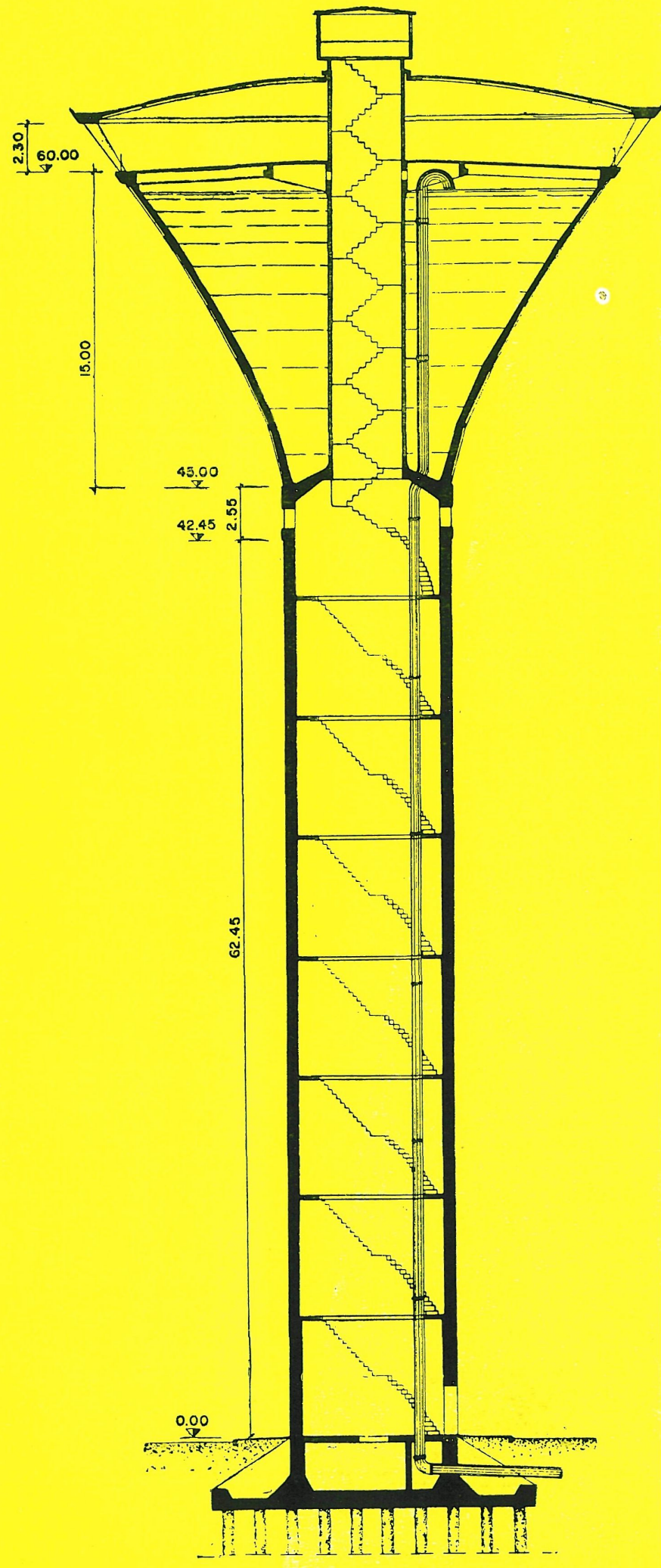

Este depósito elevado, de hormigón armado y $2.000 \mathrm{~m}^{3}$ de capacidad, tiene por objeto alimentar de agua a la factoría que la casa Fiat ha instalado en Turín (Ítaiia).

Tiene 59,50 m de altura entre el suelo y el nivel máximo superior del agua. Sobre la cubierta se ha creado una terrazamirador, desde donde se puede admirar el paisaje local y el fondo alpino, con acceso por medio de un ascensor. Sobre aquél se ha construido una cabina para li maquinaria del elevador.

La cámara de bombeo de agua se halla instalada en las proximidades de la base del fuste.

\section{Cimientos}

$\mathrm{Su}$ cimiento es una losa circular, de hormigón armado, de $15,5 \mathrm{~m}$ de diámetro. Un disco concéntrico, de $8 \mathrm{~m}$ de diámetro exterior y $80 \mathrm{~cm}$ de espesor, sobre el que se han levantado tabiques radiales empotrados en una corona de $50 \mathrm{~cm}$ de tabiques radiales empotrus cual descansa sobre 75 pilotes de $50 \mathrm{~cm}$ de diámetro y de $80 \mathrm{t}$ cual descansa sobre 75 pilotes de $50 \mathrm{~cm}$ de diámetro y de $80 \mathrm{t}$
de capacidad de sustentación cada uno, carga que, en las condiciones más desfavorables del viento, se eleva a 100 toneladas.

\section{Fustote}

La estructura soporte está constituida por un fuste de $8 \mathrm{~m}$ de diámetro, de $60 \mathrm{~cm}$ de espesor hasta la cota $5,65 \mathrm{~m}$ y de $50 \mathrm{~cm}$ hasta la cota 42,45 metros.

La superficie externa se formó con paneles prefabricados-que servirían de encofrado-de $4 \mathrm{~m}$ de radio de curvatura, $2,83 \mathrm{~m}$ de altura y un espesor de $3 \mathrm{~cm}$. Estos paneles curvados tienen una sección en forma de $U$, con alas asimetricas de $50 \mathrm{~cm}$ la mayor, lo que corresponde al espesor del fuste una vez terminado. Los paneles (20 de cada clase) se han colocado en obra uniendo entre sí sus dos al frado capaz de resistir al peso propio del hormigón fresco.

\section{sección del fuste}

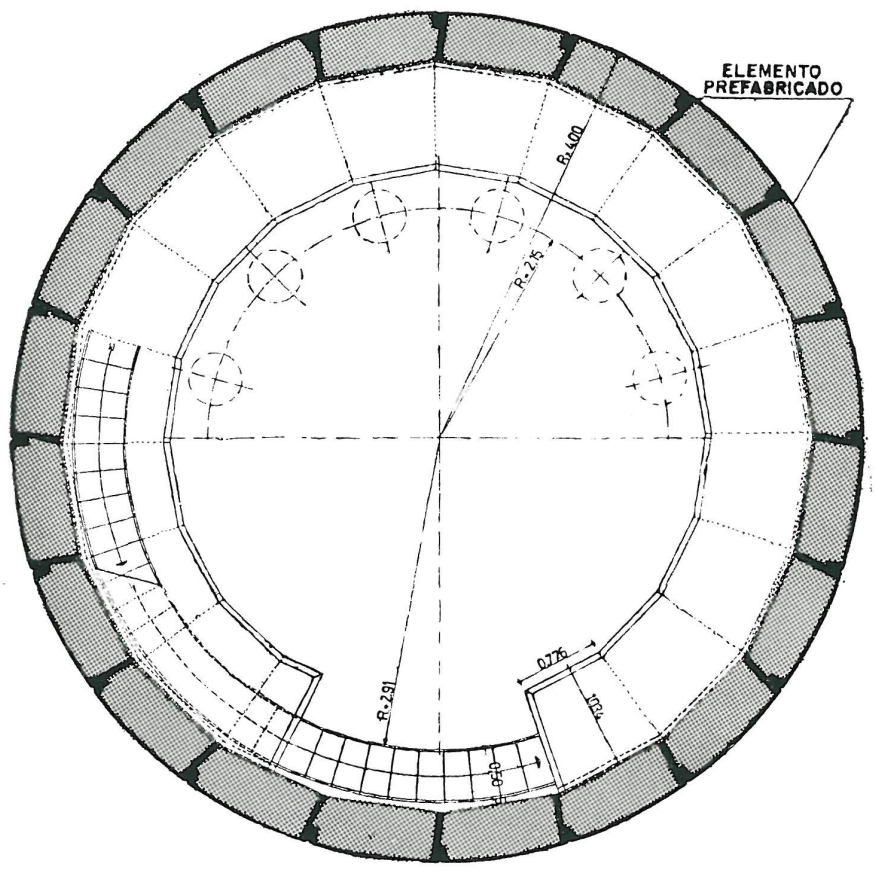




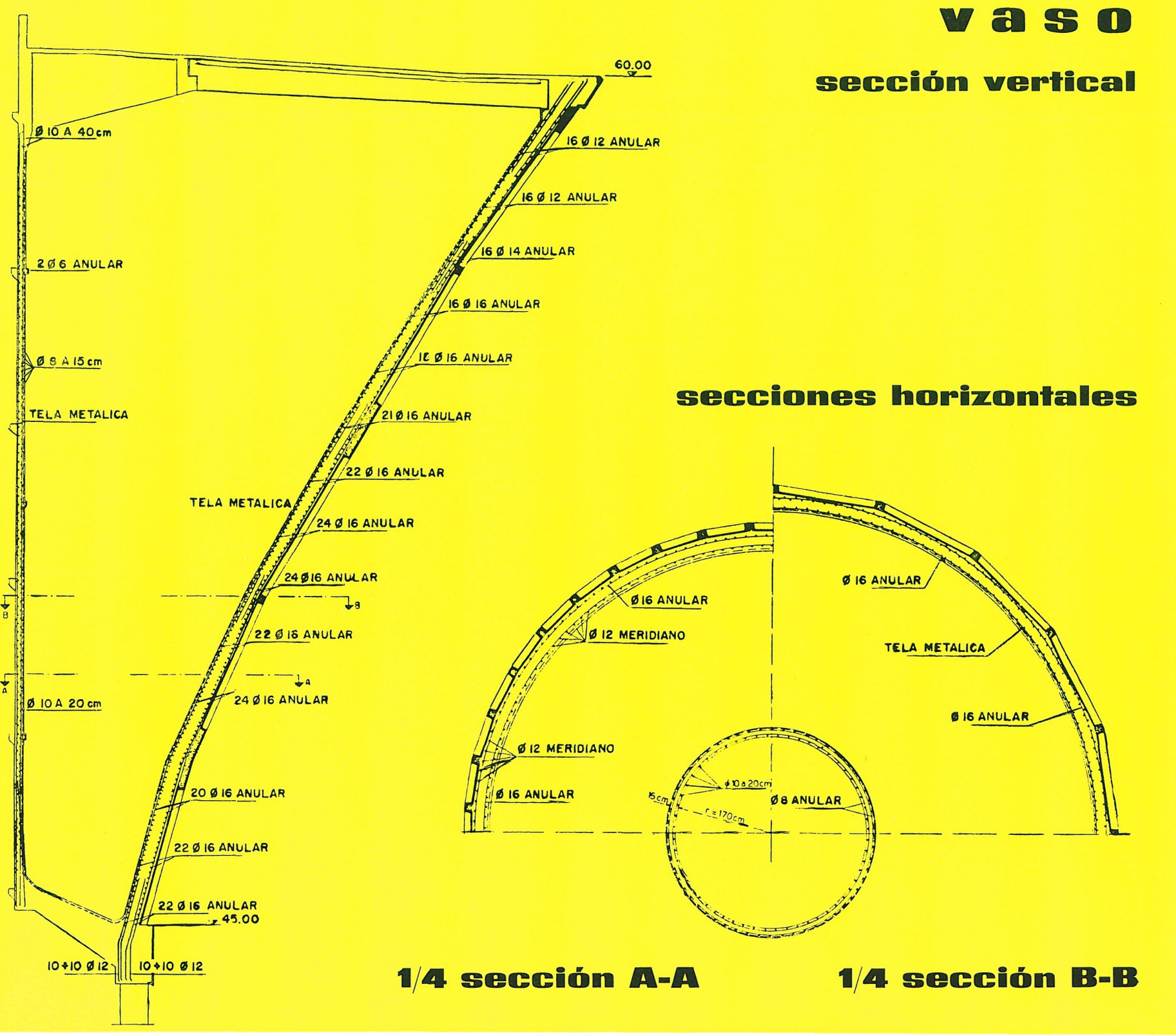

En el interior del encofrado así constituido se colocaron las armaduras verticales que se unieron a los anillos circulares de $50 \times 50 \mathrm{~cm}$ que forman las armaduras horizontales. El encofrado interior es de madera, retenido en posición con tirantes metálicos anclados en los paneles y espaciadores de hormigón para mantener el espesor previsto. Los anclajes, vistos desde el exterior, se han dispuesto ordenadamente a fin de lograr un buen aspecto estético al exterior.

Para llegar a la base del depósito propiamente dicho se necesitaron 15 clases de paneles. La puerta de acceso se consigue suprimiendo dos de estos paneles, que dejan un hueco de $2 \times 2,33 \mathrm{~m}$. Los elementos interiores se han colocado desde un balconcillo de elementos prefabricados que vuelan hacia el interior. Este balconcillo se une a las armaduras de los paneles exteriores y a los anillos interiores, formando así, después de hormigonar, un todo monolítico. Los elementos prefabricados de cada balconcillo van provistos de los huecos correspondientes para el paso de las tuberías del depósito, guías para el ascensor, montantes para el antepecho y escaleras hasta el balconcillo superior.

La parte alta del fuste consta de un anillo circular, de $50 \times 50 \mathrm{~cm}$ de sección, sobre el que se levantan veinte montantes, de $50 \times 50 \mathrm{~cm}$, y un segundo anillo, de $1 \times 0,65 \mathrm{~m}$ de sección, que sirve de apoyo al recipiente del depósito. Tanto los anillos como los montantes tienen la parte vista exterior formada por paneles de $3 \mathrm{~cm}$ de espesor.

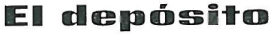

El recipiente tiene una superficie externa de revolución, de sección meridiana parabólica, fondo troncocónico y un núcleo central cilíndrico, hueco, para el paso de la escalera de servicio y el ascensor. 


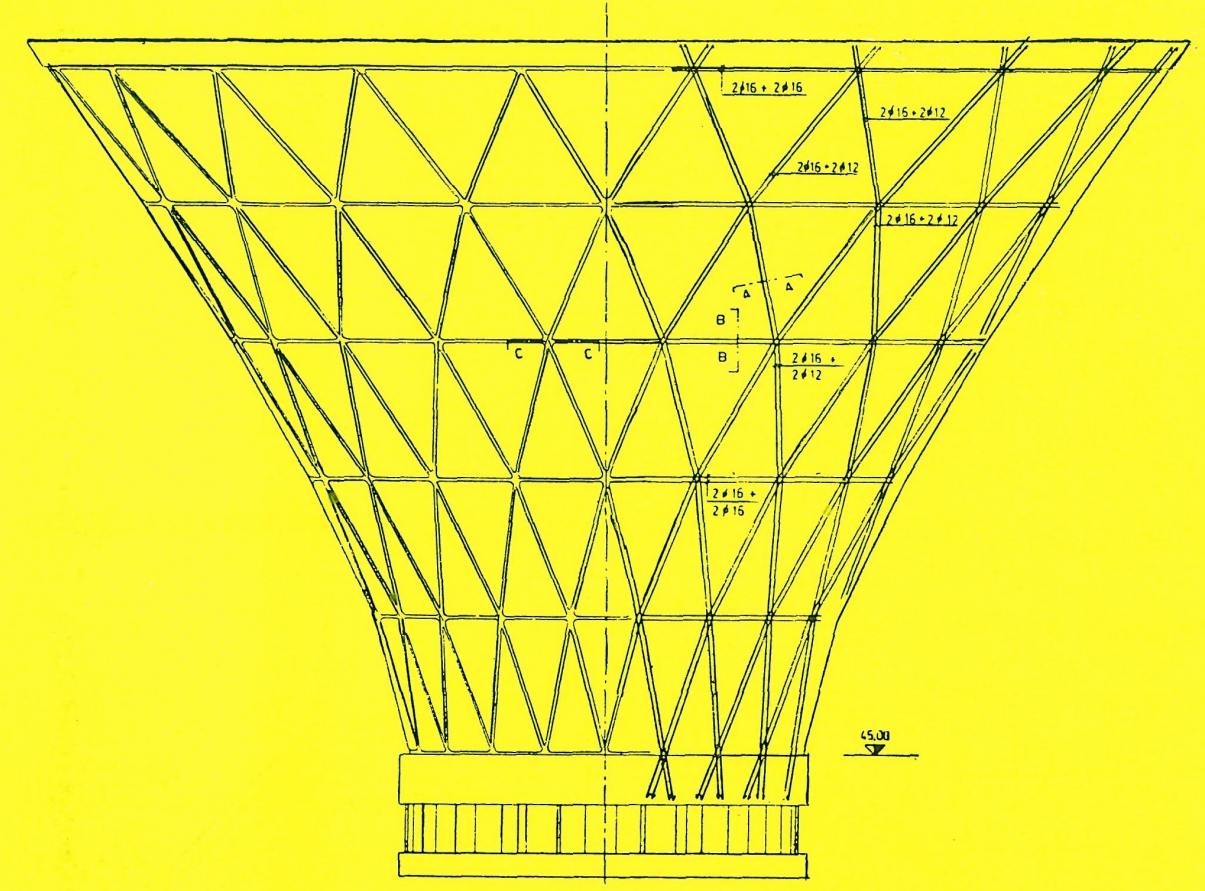

alzado del vaso

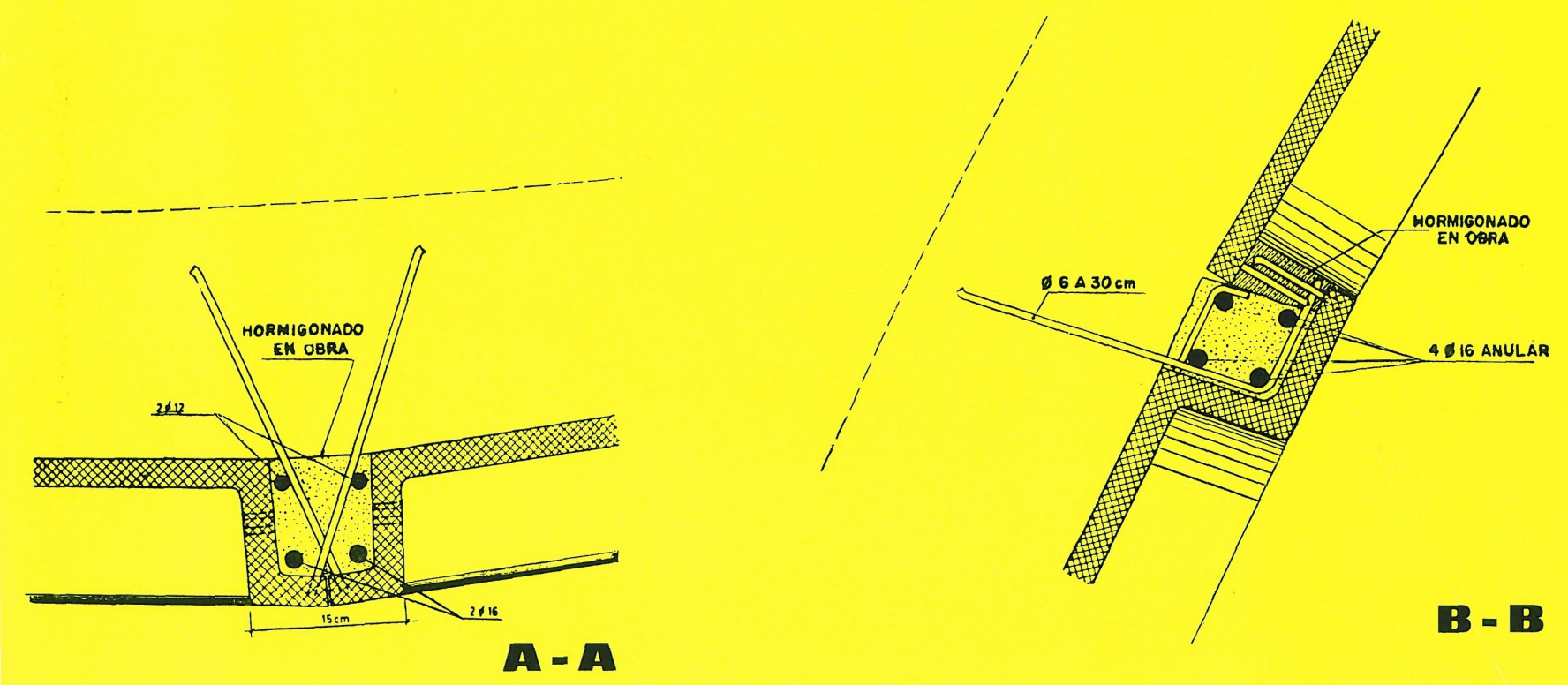

\section{detalles}

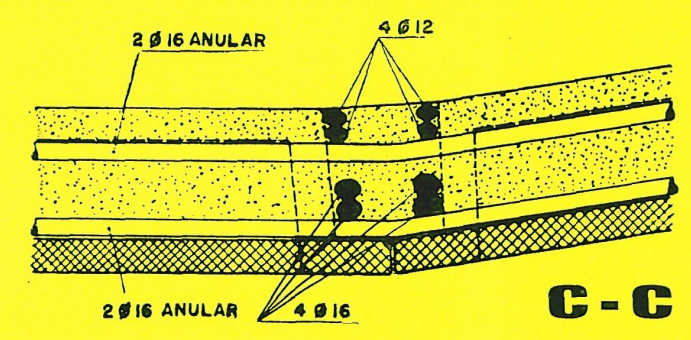




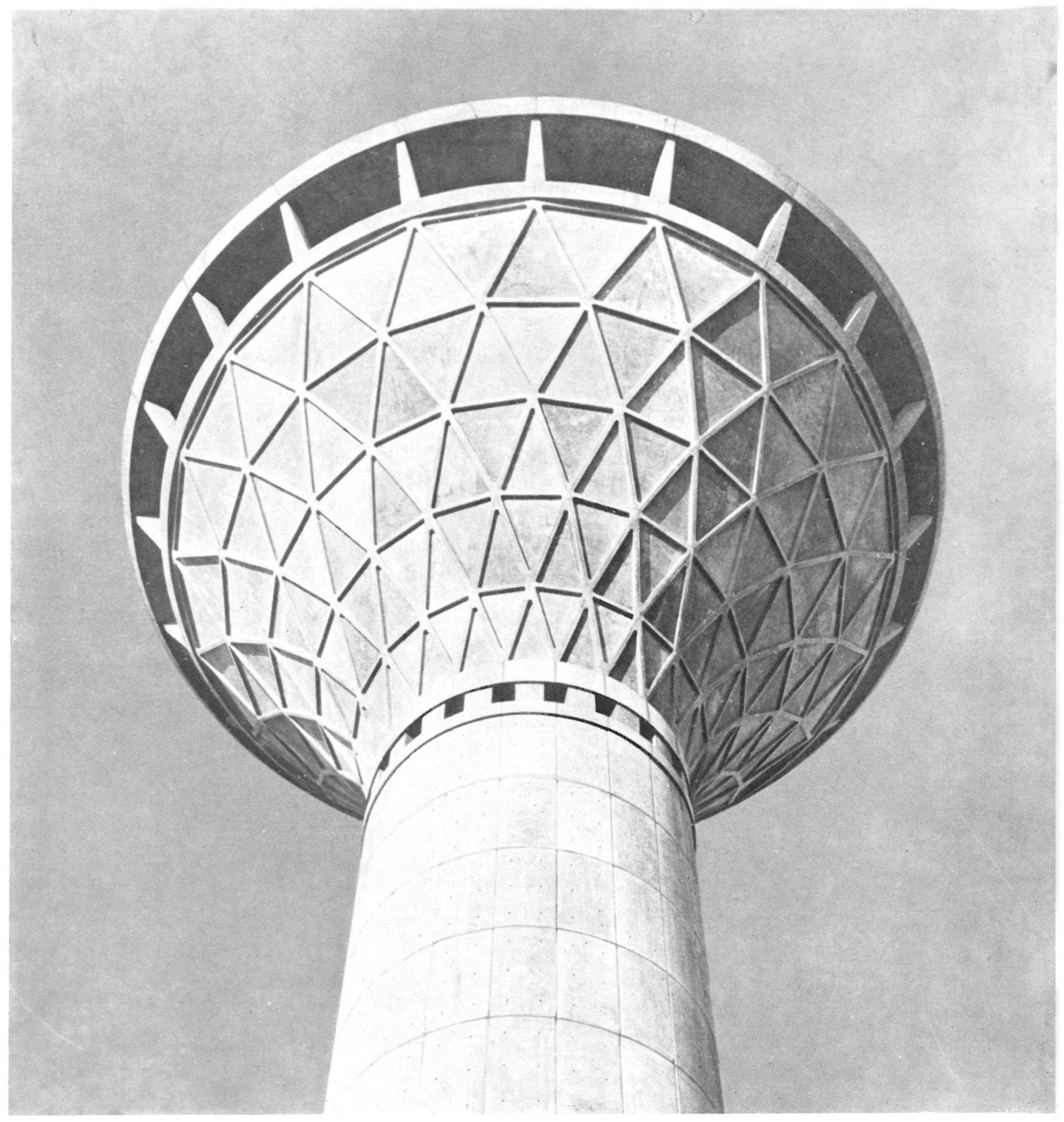

Cubeta y mirador.

La pared exterior está formada por cinco series de 40 paneles triangulares, los cuales se colocaron apoyándose en un entramado tubular, en voladizo, y hormigonando las juntas de forma tal que quedase un nervio en todo el perimetro de cada panel, con lo que se consiguio lograr suficiente resistencia para que los anillos troncocónicos así formados pudieran soportar el peso del hormigón fresco de la pared. El entramado tubular que rodea al fuste se prolongó, en su parte superior, en voladizo, para servir de apoyo durante la colocación de los paneles de la cubeta del depósito.

Después del hormigonado de nervios y de la colocación de armaduras, se procedió a hormigonar la pared sin encofrados interiores, lo cual se consiguió utilizando cuatro capas de tela metálica que retenían el hormigón fresco, permitían su control y evitaban la formación de huecos, dejando una superficie rugosa ideal para extender sobre ella un enfoscado final. Estas cuatro capas de tela metálica se colocaron sobre las armaduras anulares y meridianas de la pared, fijánnen bien al hormigón fresco, aun vibrado enérgicamente. Las armaduras anulares se repartieron apropiadanen bien al hormigón fresco, aun vibrado enérgicamente. Las armaduras anulares se repartieron apropiada-
mente para resistir al empuje del agua. El espesor de la pared varía de $35 \mathrm{~cm}$ en la base a $20 \mathrm{~cm}$ en su parte media. Las tensiones de tracción del hormigón se mantienen siempre inferiores a $20 \mathrm{~kg} / \mathrm{cm}^{2}$.

La estructura que cubre la cubeta, sirviendo de forjado al mirador, se calculó para una sobrecarga de 350 kilogramos $/ \mathrm{cm}^{2}$. La superficie interior del depósito se trató con varias capas de impermeabilización. 


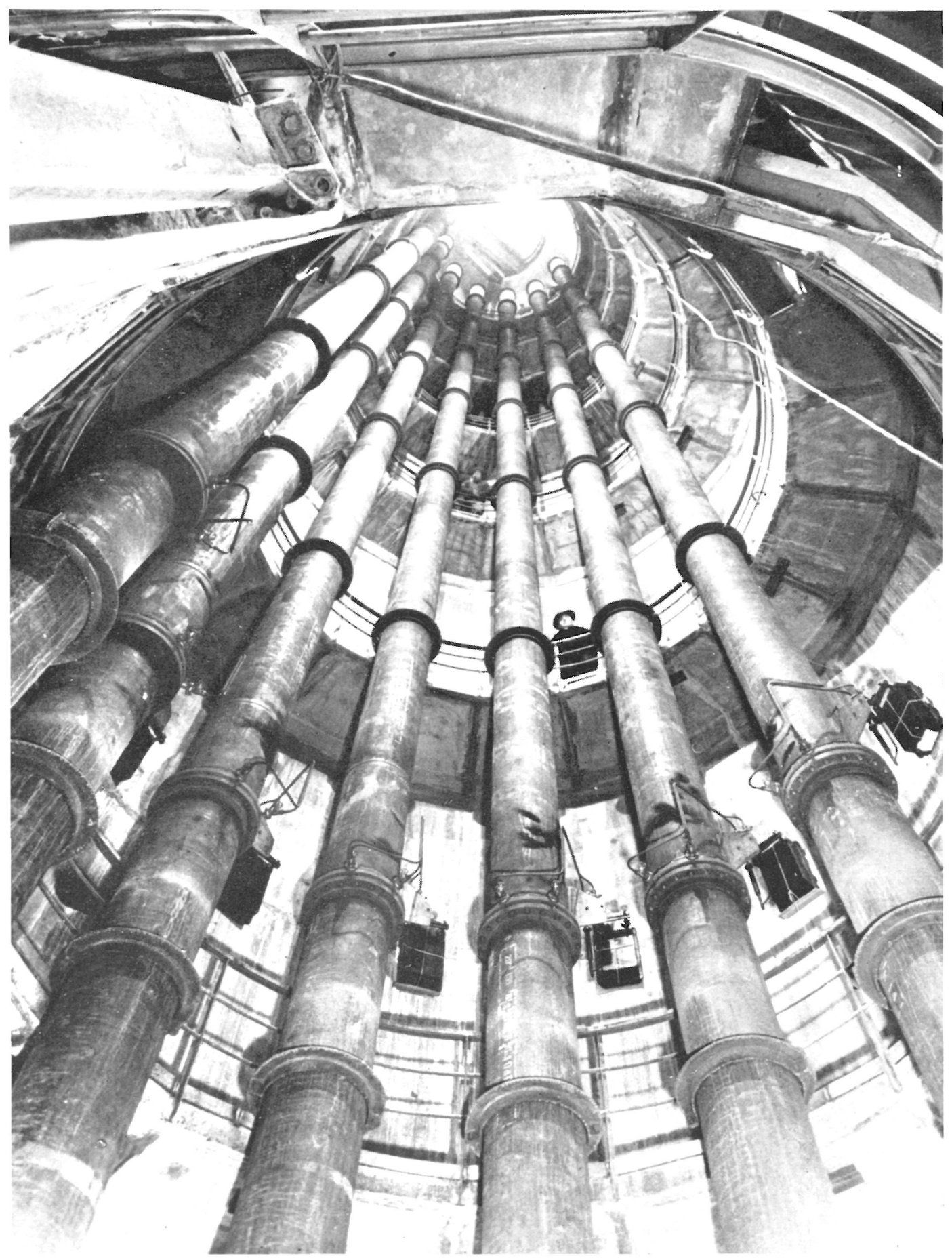

Vista del interior del fuste.

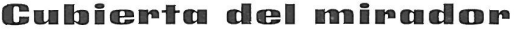

Está cubierto con una cúpula esférica, de $25 \mathrm{~m}$ de diámetro, soportada por veinte montantes de $2,30 \mathrm{~m}$ de altura, los cuales han sido estudiados para permitir movimientos de rótula en sus bases y de dirección radial, con objeto de absorber cualquier esfuerzo provocado por la bóveda, como consecuencia de dilataciones posibles, sin que por ello se transmitan momentos flectores al anillo-imposta de la pared de la cubeta. La dilatación del anillo de la cúpula puede ser distinta a la del anillo superior de la cubeta, ya que ésta, debido a su contacto con el agua, tiene una temperatura mucho más regular. La cúpula está constituida por paneles prefabricados, de curvatura esférica y de $2,5 \mathrm{~cm}$ de espesor, rigidizados con nervios meridianos. Sobre los paneles se colocó una losa de $4 \mathrm{~cm}$ de espesor. En la zona de unión entre cúpula y fuste cilíndrico prolongado se ha formado una junta de dilatación que permite el juego de las distintas dilataciones. Sobre la cúpula va la cabina, de estructura mepermite el juego de las distintas dilatacion
tálica, para la maquinaria del ascensor. 


\section{elemento prefabricado del fuste}

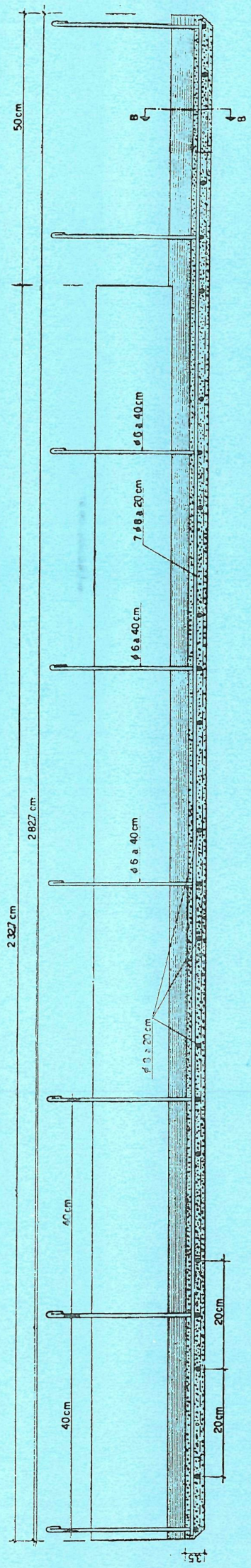

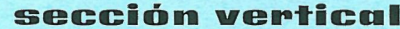

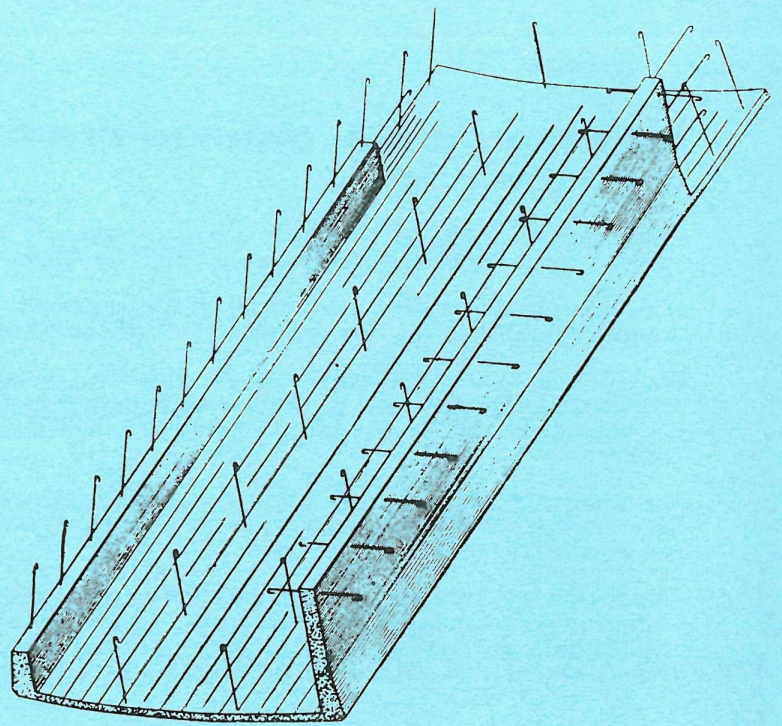

perspectrive axnmétrica de un elemento
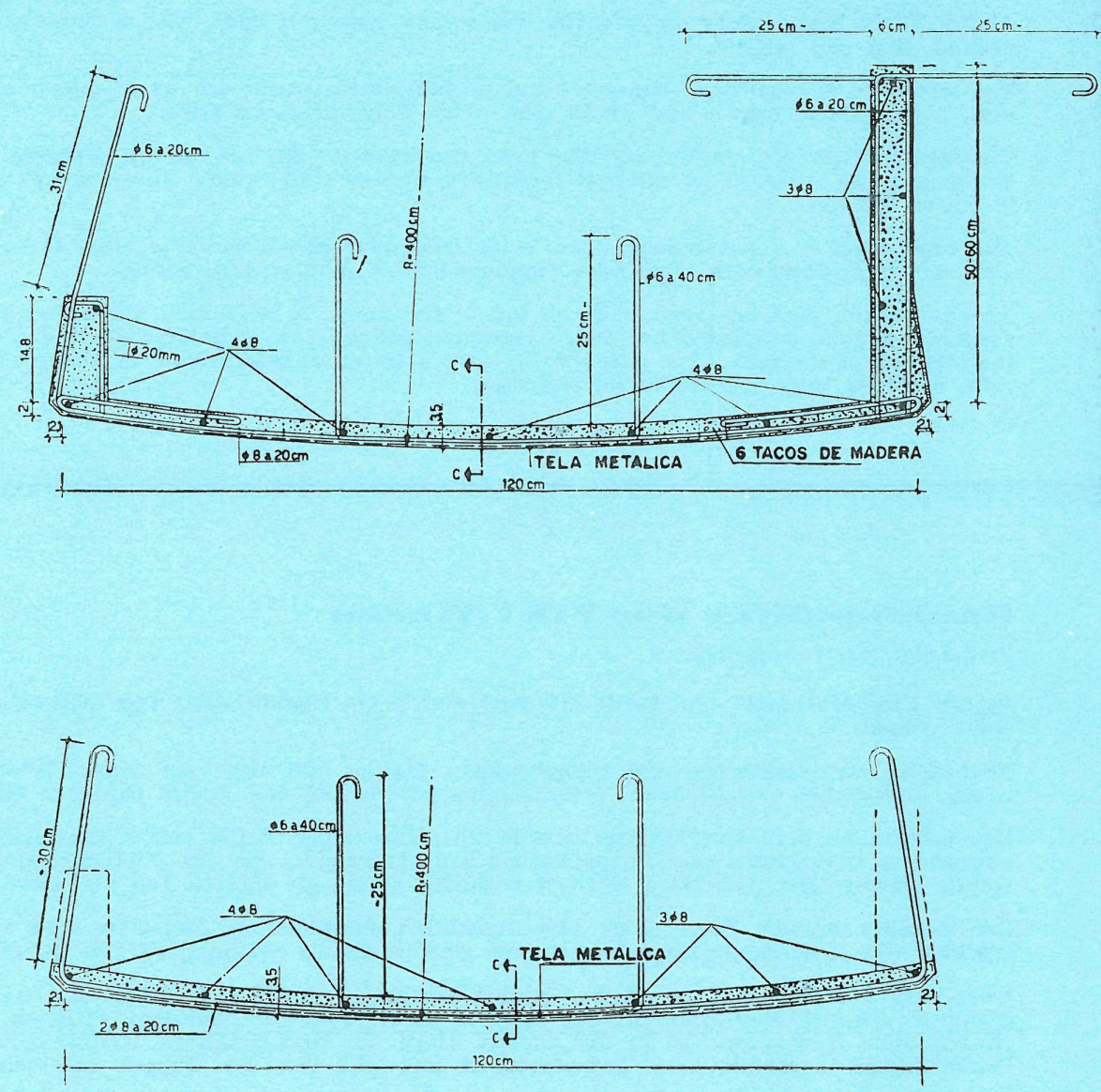

secciones thorizontalles 


\section{Châtean d"eau pour Ia IFIAT}

Pier Luigi Nervi, ingénieur.

Un château d'eau, en forme de verre à pied, de $2.000 \mathrm{~m}^{3}$ de capacité et d'environ $60 \mathrm{~m}$ de hauteur au-dessus du sol, a été construit pour l'usine FIAT de Turin (Italie).

Ce château d'eau de béton armé et de panneaux préfabriqués, s'appuie sur un disque de béton, une couronne cylindrique et des contreforts radiaux, l'ensemble reposant sur des pilotis.

Le fût, de $8 \mathrm{~m}$ de diamètre, a été construit à l'aide d'une série de panneaux, de surface cylindrique et de section transversale en U, d'ailes asymétriques, de $3 \mathrm{~cm}$ d'épaisseur, qui ont servi de coffrage extérieur pour le bétonnage des murs, dont l'épaisseur varie de $60 \mathrm{~cm}$ dans la zone inférieure à $50 \mathrm{~cm}$ dans la zone supérieure.

Le réservoir est une méridienne parabolique de révolution, constituée par des panneuax triangulaires, formant des anneaux tronco-coniques, qui ont été utilisés également comme coffrage, appuyé sur un treillis tubulaire en encorbellement.

Au-dessus du réservoir a été construite une terrasse de $25 \mathrm{~m}$ de diamètre, couverte par une coupole nervurée sphérique, supportée par des montants articulés qui permettent des mouvement radiaux sans que des moments fléchissants se transmettent à l'anneau supérieure du réservoir qui, étant plein d'eau, a des températures plus régulières et une dilatation différente de celle de la coupole.

\section{Elewated tramk for the Firat Motromwolks}

Pier Luigi Nervi, architect.

At the Turin Fiat factory an elevated water tank has been built, with a capacity of $2,000 \mathrm{~m}^{3}, 60 \mathrm{~m}$ above the ground, and cup shaped.

The structure is reinforced concrete, and prefabricated panels are used, which are supported by a concrete disc, a cylindrical crown and radial ribs. The whole rests on piles.

The stem is $8 \mathrm{~m}$ in diameter, and has been constructed with the aid of $U$ shaped cylindrical panels, $3 \mathrm{~cm}$ in thickness, which served as external formwork for the stem walls. These are $60 \mathrm{~cm}$ thick at the bottom, and $50 \mathrm{~cm}$ at the top.

The tank itself is a paraboloid, made with triangular panels which together form conical circular rings. These also served as formwork, and were supported by a tubular scaffolding.

An observation post has been built on top of the tank. It is $25 \mathrm{~m}$ in diameter, and is covered with a ribbed spherical cupola, supported by hinged columns, which allow radial deformations, which are not transmitted to the top ring of the tank. The latter, being full of water, is kept at a more uniform temperature than the cupular roof, which is fully exposed to the weather.

\section{Hochlbehäilter der FIAT-Wemke}

Pier Luigi Nervi, Ingenieur.

In der FIAT-Werkstatt von Turin (Italien) wurde ein Hochbehälter von $2.000 \mathrm{~m}^{3}$ Fassungsvermögen und $60 \mathrm{~m}$ Höhe gebaut.

Er besteht aus Stahlbeton und vorgefertigten Platten und liegt auf einer Betonplatte, einem zylindrischen Kranz und radial verlaufenden Verstärkungspfeilern auf. Das Ganze ruht auf Säulen.

Der Schaft von $8 \mathrm{~m}$ Durchmesser wurde mit Hilfe einer Reihe von $3 \mathrm{~cm}$ dicken zylindrischen Platten mit u-förmigem Querschnitt und asymetrischen Flügeln gebaut, die als Aussenschalung für das Betonieren der Wände dienten. Die Wanddicke variiert zwischen $60 \mathrm{~cm}$ im unteren Teil und $50 \mathrm{~cm}$ im oberen Teil.

Der Behälter hat die Form eines sich drehenden parabolischen Meridians und wird durch dreieckige Platten gebildet, die Kegelstumpfringe bilden und gleichzeitig als Schalung auf ein Rohrkraggerüst gestützt dienen.

Über dem Behälter hat man einen Ausblick geschaffen, der $25 \mathrm{~m}$ Durchmesser hat und von einer kugelförmigen gerippten Kuppel überdacht ist. Die Kuppel wird durch Gelenkstützen getragen, die radiale Momente ermöglichen ohne Biegemomente an die oberen Ringe des Hochbehälters weiterzuleiten. Dieser hat durch seine Wasserfüllung regelmässigere Temperaturen und eine von der Kuppel unterschiedliche Ausdehnung. 